\title{
Switching device based on the quantum Hall effect
}

R. F. Kazarinov

Serge Luryi

Bell Laboratories

Murray Hill, New Jersey 07974

\section{ABSTRACT}

Novel quantum device - a voltage amplifier - is

proposed, based on controlled phase transition

of percolation type in semiconductor inversion

layers in strong transverse magnetic field.

The quantized Hall effect (QHE) is a novel macroscopic quantum phenomenon recently discovered $[1,2]$ in semiconductor inversion layers at liquid helium temperatures and strong transverse magnetic fields $(B \sim 10 T)$. It is characterised by the existence of certain "plateau" values of the field $B$ and the electron surface density $\sigma$ in the inversion layer. In these plateaus the transverse (Hall) conductivity is exactly $\sigma_{x y}=\bar{n} e^{2} / h$, with $\bar{n}$ integer, while the longitudinal conductivity $\sigma_{x x}$ and resistance $\rho_{x x}=\sigma_{x x} /\left(\sigma_{x x}^{2}+\sigma_{x y}^{2}\right)$ both nearly vanish. The QHE has been used for high-accuracy measurements of the ratio $e^{2} / h$ and the fine structure constant.
To be specific we consider the heterojunction realization [2] of the QHE, in which the inversion layer is formed on the p-GaAs/n-GaAlAs interface due to a work function difference, cf. Fig.1. At low temperatures only the lowest energy subband in the quantum well is occupied. In strong magnetic fields this 2-dimensional subband, corresponding to the electron motion in the plane of the inversion layer (normal to $B$ ),

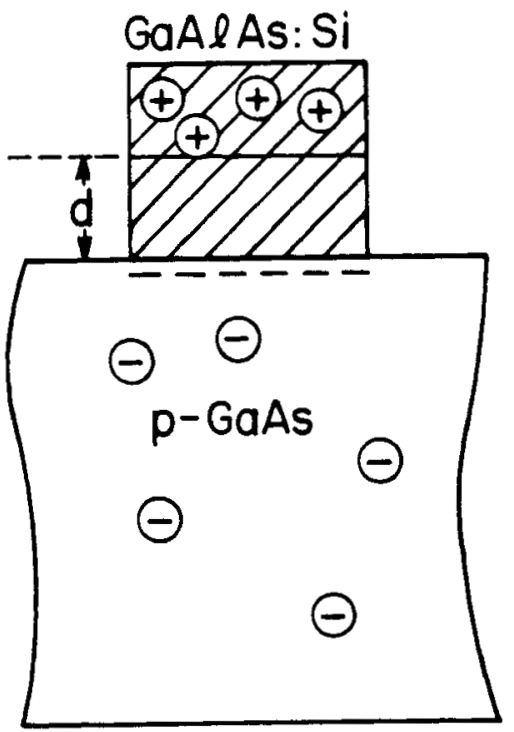

Figure 1. Cross-section of the sample studied in ref. 2.

\section{7}


is split into discrete Landau levels, $E_{n}=(n+1 / 2) \hbar \omega$, where $\omega=e B / m c$ is the cyclotron frequency, and $m$ is the effective mass

In an ideal system with no disorder, each Landau level is multiply degenerate, containing $e B / h c$ states per unit area (for $B=10 T$ this gives, approximately, $3 \times 10^{11}$ states $/ \mathrm{cm}^{2}$ ). If there is an electric field in the plane of the inversion layer, then this degeneracy is lifted. The resultant electronic states are localized in the direction of the (local) electric field by the magnetic length $a=(\hbar c / e B)^{1 / 2}($ for $B=10 T, a \approx 70 \AA)$ In the direction of the magnetic field the states are, of course, also localized by the quantum well, while in the third direction the states are delocalized. The electronic waves propagate along the electrostatic equipotential lines like light in an optical fiber.

It has been shown $[3,4]$ that the QHE is a manifestation of long-range coherence of electronic states. In a Corbino geometry, Fig. 2, the QHE implies the existence of electron quantum orbits extending around the entire ring like in a gigantic atom. For these global fibers to exist it is necessary that there must be global equipotential lines within the plane of the inversion layer. The fact that such lines do exist in a macroscopic sample is rather surprising, given the unavoidable disorder of the electrostatic potential. In a heterojunction QHE sample this disorder is brought about by random variations in the surface density of the fixed charge responsible for the creation of the inversion layer.

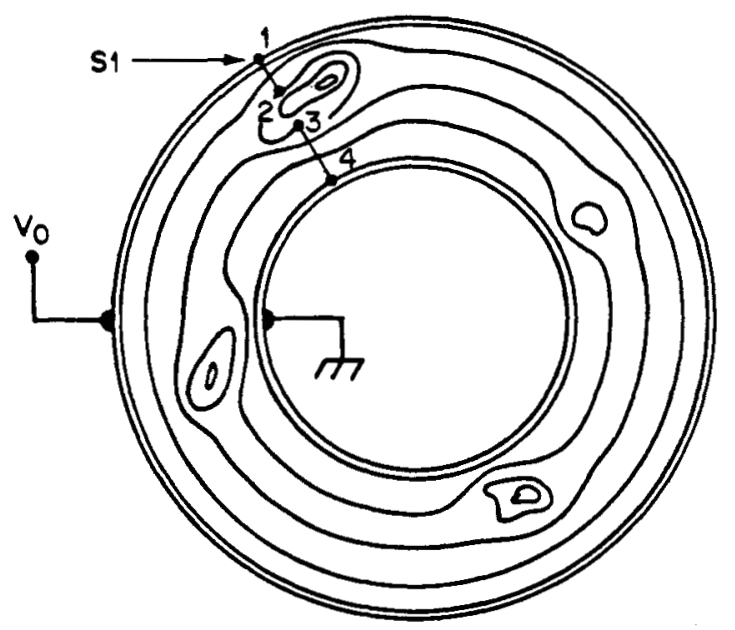

Figure 2. Schematic diagram of the local and global fibers.

Most of electronic states in the sample represent extended but local fibers revolving around potential hills or cavities, see Fig. 2. It has been shown [5] that in equilibrium all states are local, while global fibers begin to appear only when the Hall voltage exceeds a certain critical value $V_{c r}$. By the order of magnitude, $V_{c r}$ equals the characteristic value of the potential fluctuations on the scale of the sample size. If one can imagine turning on the disorder at a given applied Hall voltage, then at some point one would 
eliminate all the global equipotentials and the Hall current would stop abruptly. This phenomenon can be interpreted as a phase transition of percolation type, in which the Hall current plays the role of an order parameter

A remarkable property of the described percolation transition is that it can be brought about in a controlled fashion by simulating "disorder" with the help of a voltage applied to a gate. An entirely new quantum device can be based on this principle. The proposed device, called the PHASER (the name stems from two sources: the controlled phase transition and the modulation of electronic phase), is shown in Fig.3. It represents a Corbino disk with two pairs of contacts to the inversion

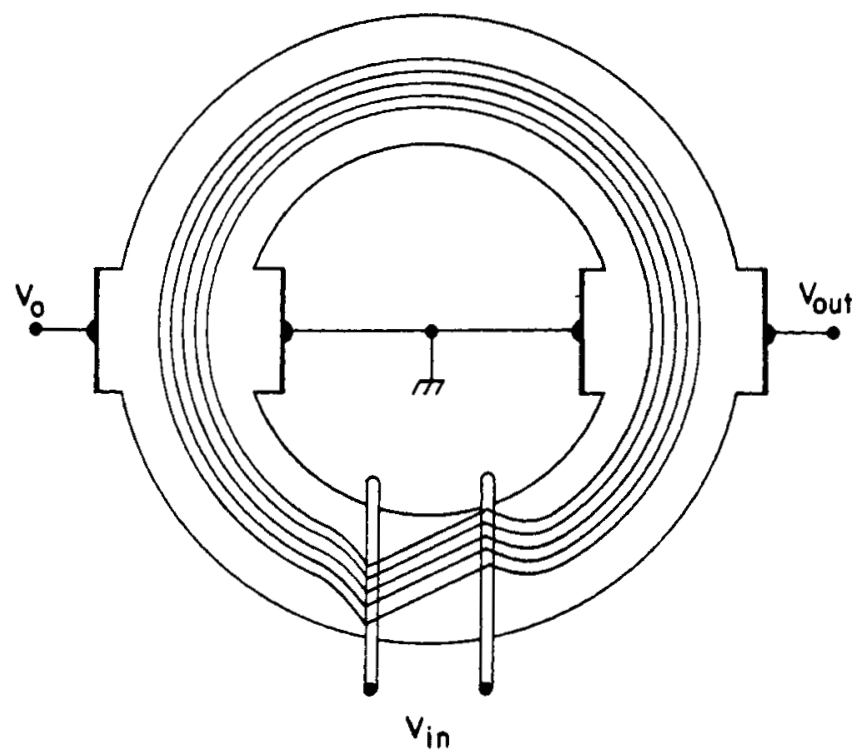

Figure 3. The PHASER. Schematic layout of the device, showing global fibers at a finite input voltage. layer and two parallel insulated gate electrodes deposited on the surface of the disk. We shall analyze the PHASER operation for an ideal case with no random potential. In the absence of the gate voltage the electron wave-guides (quantized equipotential lines) represent global fibers and the Hall current is flowing. In this case the output voltage $V_{\text {out }}$ exactly equals the applied voltage $V_{0}$. This result is unaffected by the presence of local states. Indeed, consider a radial section of the sample which crosses one or more closed loops, e.g., section $\mathrm{S} 1$ in Fig. 2. Because points 2 and 3 lie on an equipotential, the sum of Hall voltages developing in regions $1-2$ and 3-4, equals the applied voltage $V_{0}$. In other words, when a quantum Hall current is flowing in the Corbino ring, the latter represents an ideal voltage transducer, since the Hall e.m.f. in every section of the ring equals the applied voltage. However, when the quantum coherence is broken by the gate field, then the Hall e.m.f., which is our output voltage, drops to zero.

When an input voltage $V_{\text {in }}$ is applied between the gate electrodes the shape of the equipotential lines changes as shown in Fig.3. The blank area is bounded by a local (though long!) equipotential fiber. Electrons within this area have insufficient energy to pass over the potential barrier created by the gate and suffer a quantum mechanical reflection. Two kinks shown on the global 
fibers correspond to a refraction of electron waves under the gate electrode. The number of global fibers is reduced but the Hall current and therefore $V_{\text {out }}$ remain constant. This is equivalent to an effective reduction of the Corbino disk width. When the gate voltage $V_{\text {in }}=V_{0}$ the effective disk width vanishes which is equivalent to the phase transition described above. The ring Hall current ceases abruptly and so does the Hall e.m.f. in the output circuit. In this state $V_{\text {out }}=0$. The resulting transfer characteristic of a PHASER is shown in Fig.4.

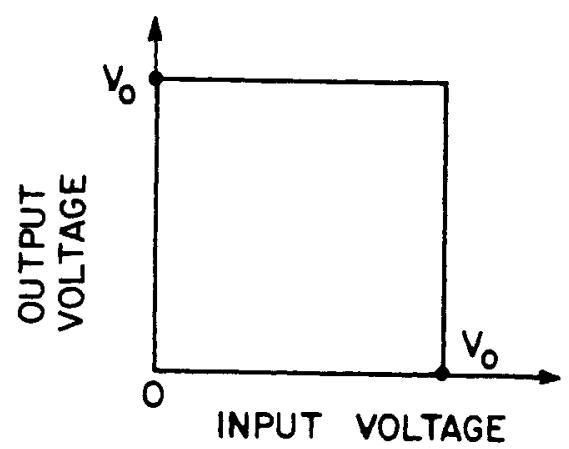

Figure 4. Transfer characteristic of a PHASER.

Even though an insulated gate is involved the principle of operation of the PHASER is different from any field-effect device in that the surface density of electrons in the channel remains constant during switching. To illustrate this point we consider a capacitor formed by the 2-d EG and an insulated gate. In a strong magnetic field the differential capacitance, $C=\partial \sigma / \partial V_{G}$, is "quantized", vanishing in the plateau regions. This effect has been observed [6], its existence follows straightforwardly from the existence of gaps in the density of states spectrum. In a $C=0$ plateau the inversion layer behaves like an insulator with respect to the transverse field so long as the applied voltage is insufficient to transfer an electron from the gate (Fermi level) to the next unfilled Landau level.

Ideally, the speed of a PHASER is limited only by the electron inertia, i.e., the cyclotron frequency $\omega$. We should emphasize that this estimate applies only to a hypothetical device with no disorder, and no external parasitic capacitance. In such a device the energy required to switch on or off the Hall current is associated only with the kinetic energy of Hall electrons, which can be typically of order $10^{-20} \mathrm{~J}$. In practice, however, there are unavoidable parasitic capacitances, both external and internal, associated with the localized states. In strong magnetic field, all these capacitances are charged through the Hall resistance, $R_{x y}=h / \bar{n}^{2} \approx 25 k \Omega / \bar{n} . \quad$ This should severely limit the speed of operation and, probably, render the PHASER concept impractical.

Acknow ledgements

It is our pleasure to thank M.Gurvitch, 
C.H.Henry, J.C.Hensel, and H.L.Störmer for helpful comments.

References

[1] K. von Klitzing, G. Dorda, and $M$. Pepper, Phys. Rev. Letters 45, 494 (1980).

[2] D. C. Tsui and A. C. Gossard, Appl. Phys. Letters 38, 550 (1981).

[3] R. B. Laughlin, Phys. Rev. B23, 5632 (1981).

[4] R. F. Kazarinov and Serge Luryi, Phys. Rev. B25, 7626 (1982).

[5] Serge Luryi and Rudolf F. Kazarinov, to be published.

[6] M. Kaplit and J. N. Zemel, Phys. Rev. Letters 21, 212 (1968). 